\title{
Sliding Mode-Based Control of a UAV Quadrotor for Suppressing the Cable-Suspended Payload Vibration
}

\author{
Tom Kusznir (i) and Jaroslaw Smoczek iD \\ AGH University of Science and Technology, Faculty of Mechanical Engineering and Robotics, Krakow 30059, Poland \\ Correspondence should be addressed to Jaroslaw Smoczek; smoczek@agh.edu.pl
}

Received 11 September 2019; Revised 26 November 2019; Accepted 17 December 2019; Published 11 January 2020

Academic Editor: Radek Matušů

Copyright ( $\odot 2020$ Tom Kusznir and Jaroslaw Smoczek. This is an open access article distributed under the Creative Commons Attribution License, which permits unrestricted use, distribution, and reproduction in any medium, provided the original work is properly cited.

\begin{abstract}
This paper addresses the problem of damping vibrations of a cable-suspended payload during positioning of the quadrotor. A nonlinear model is derived for the coupled quadrotor-pendulum system in the $X-Z$ plane using Euler-Lagrange formulation. Sliding mode control (SMC) is used for horizontal positioning and payload vibration damping, while a feedback linearizing controller is used for both altitude and attitude control. The SMC surface parameters are determined by placing the eigenvalues of the linearized system at a desired position. The simulation results show the effectiveness of the proposed control method in minimizing payload vibration by comparing it with a partial feedback linearizing controller and a ZVDD input shaper.
\end{abstract}

\section{Introduction}

During the last decade, technological advancements in unmanned aerial vehicles (UAVs) have increased significantly. This has led to a wide range of applications such as highly advanced UAVs developed for military purposes; commercial UAVs used for cinematography, surveying, inspection, etc.; and UAVs developed by academic institutions for research into robotics, control systems, etc. [1]. One of the main applications used by all three, military, commercial, and academic, UAVs is that of load transportation. Load transportation has long been used in manned aerial vehicles, such as helicopters, with applications including heli-logging, to obtain timber from hard to reach or environmentally sensitive areas, or during search and rescue operations. Currently, aerial load transportation with small UAVs is being used in tasks such as construction, disaster response, package delivery, agriculture, military scenarios, and search and rescue missions [2-4].

The two most common ways of aerial transportation are by either using a robotic arm with a gripping mechanism or by suspending the payload with a cable attached to the UAV [5]. The robotic arm approach has several drawbacks including increased weight, varying inertia, which reduces maneuverability, and increased costs [6]. In contrast to the robotic arm, a cable-suspended payload does not have the added mechanical complexity, but it introduces a new set of challenges such as transient and residual vibrations of the cable. The transient vibrations pose a safety hazard to the $\mathrm{UAV}$, to the objects within the workspace of the UAV, and to the payload itself, while the residual vibrations increase the time to position the payload accurately.

Quadrotors, although being a highly nonlinear, coupled, underactuated, and unstable system, have become one of the most widely used vertical take-off landing (VTOL) UAVs due to their mechanical simplicity. By attaching a payload suspended with a cable to the center of mass of the quadrotor, an extra degree of underactuation is added to the system in planar motion which adds additional complexity. The cable-suspended load is a hybrid system in which the two states are determined by the tension in the cable, being either taut or slack [7].

Researchers have tackled the problem of reducing residual vibrations with various open-loop and feedback control strategies. The most common open-loop methods in payload vibration suppression during transportation are input command shaping and trajectory optimization. Input command shaping has a long history in suppressing 
vibrations in systems such as cranes [8-10]. In [11], a combination of a feedforward ZVD shaper to reduce payload oscillations was used together with a feedback modelbased controller. In [12], the authors implemented input command shaping for vibration suppression in conjunction with a feedback linearizing controller for the quadrotor. Trajectory optimization is an optimal control technique that requires the desired state trajectories be calculated beforehand which is usually computationally expensive. In [6], the authors optimized the trajectory to produce energy efficient maneuvers by reformulating the trajectory planning problem as a Mathematical Program with Complementary Constraints (MCPP) and solving it using Sequential Quadratic Programming (SQP). In [13, 14], the authors use dynamic programming for trajectory optimization that suppresses oscillations for the quadrotor with a cable-suspended payload.

Feedback control strategies that were used for reducing payload vibrations during transportation include those in [15] where a discrete-time state feedback mixed $H_{2} / H_{\infty}$ was developed for tiltrotors load path tracking, while in [16], model predictive control (MPC) was used for path tracking. Geometric control is implemented in $[17,18]$ to carry the payload, while in [7], the control of lifting the payload from when the cable is slack is considered. Interconnection Damping Assignment-Passivity Based Control (IDA-PBC) was used to control the quadrotor position and damping of the oscillations in [19].

In this paper, we assume the cable carrying the payload is taut during horizontal operation of the quadrotor. The differential equations describing the motion of the coupled quadrotor-pendulum system are obtained using the Euler-Lagrange formulation for a planar model. We develop a control approach that combines feedback linearization, for the altitude and attitude control, with sliding mode control for the horizontal motion of the quadrotor and sway of the payload. To obtain the parameters of the sliding surface, a pole placement method is used on the coupled system linearized around the equilibrium point. Stability of the closed-loop system is proved using Lyapunov's theorem. To demonstrate the effectiveness of the proposed control algorithm, a ZVDD input shaper is developed. Numerical simulations are carried out for three separate cases: (i) the proposed controller is subjected to a step input function with different operating conditions, (ii) a controller is subject to a continuous input function to test the tracking performance, (iii) the proposed controller is compared with the partial feedback linearizing controller and the feedback linearizing controller combined with input shaping.

The paper is organized as follows. Section 2 develops the mathematical model of the planar quadrotor-pendulum system using the Euler-Lagrange formulism. In Section 3, the controller is developed using a feedback linearizing controller for both the altitude and attitude dynamics, while SMC is utilized for horizontal motion. In Section 4, simulation results of the proposed approach are presented and compared with the partial feedback linearizing controller and the feedback linearizing controller combined with input shaping. Lastly, the conclusions are presented in Section 5.

\section{Dynamic Model}

A planar model of a quadrotor UAV with a cable-suspended payload, as shown in Figure 1, is considered. The system is modeled under assumption that the quadrotor is rigid and symmetrical and the unactuated pendulum is a point mass suspended at the end of a massless rigid rod attached to the quadrotor's center of gravity. The symbols in Figure 1 are described as the UAV quadrotor mass $M$, pendulum mass $m$, rope length $l$, pitch and payload swing angle $\theta$ and $\alpha$, respectively, and $r$ is the distance between rotors and the center mass of the quadrotor.

The generalized coordinates defining the configuration of the system are $q=[x, z, \alpha, \theta]^{T}$, where $x$ and $z$ are the positions of the center mass of the quadrotor in the $X-Z$ plane. Denoting $x_{p}=x+l \sin \alpha$ and $z_{p}=z-l \cos \alpha$ as the payload coordinates, the kinetic $K(q, \dot{q})$ and potential $U(q)$ energy of the system are

$$
\begin{aligned}
K(q, \dot{q}) & =\frac{1}{2} M \dot{x}^{2}+\frac{1}{2} M \dot{z}^{2}+\frac{1}{2} J_{\theta} \dot{\theta}^{2}+\frac{1}{2} m \dot{x}_{p}^{2}+\frac{1}{2} m \dot{z}_{p}^{2}, \\
U(q) & =M g z+m g z_{p},
\end{aligned}
$$

where $J_{\theta}$ is the inertia moment of the quadrotor and $g$ is the gravitational acceleration.

For the Lagrangian function $L(q, \dot{q})=K(q, \dot{q})-U(q)$, the system's motion equations are derived using the EulerLagrange's equation:

$$
\frac{\mathrm{d}}{\mathrm{d} t}\left(\frac{\partial L}{\partial \dot{q}}\right)-\frac{\partial L}{\partial q}=u
$$

where the input vector is given by $u=[f \sin \theta, f \cos \theta, 0, \tau]^{T}$ with the total thrust $f=f_{1}+f_{2}$ and the pitch moment $\tau=$ $r\left(f_{1}-f_{2}\right)$ provided by the rotors.

The motion equations derived from (2) are as follows:

$$
\begin{aligned}
f \sin \theta & =(M+m) \ddot{x}+m l \cos \alpha \ddot{\alpha}-m l \dot{\alpha}^{2} \sin \alpha, \\
f \cos \theta & =(M+m) \ddot{z}+m l \sin \alpha \ddot{\alpha}+m l \dot{\alpha}^{2} \cos \alpha+(M+m) g, \\
0 & =m l^{2} \ddot{\alpha}+m l \ddot{x} \cos \alpha+m l \ddot{z} \sin \alpha+m l g \sin \alpha, \tau=J_{\theta} \ddot{\theta} .
\end{aligned}
$$

Furthermore, equation (3) can be rewritten to

$$
\begin{aligned}
\ddot{x} & =\frac{M+m \cos ^{2} \alpha}{M(M+m)} f \sin \theta+\frac{m \sin \alpha \cos \alpha}{M(M+m)} f \cos \theta+\frac{m l \dot{\alpha}^{2} \sin \alpha}{M+m}, \\
\ddot{z} & =\frac{m \sin \alpha \cos \alpha}{M(M+m)} f \sin \theta+\frac{M+m \sin ^{2} \alpha}{M(M+m)} f \cos \theta-\frac{m l \dot{\alpha}^{2} \cos \alpha}{M+m}-g, \\
\ddot{\alpha} & =-\frac{\cos \alpha}{M l} f \sin \theta-\frac{\sin \alpha}{M l} f \cos \theta, \\
\ddot{\theta} & =\frac{\tau}{J_{\theta}} .
\end{aligned}
$$

Assuming that the pitch angle is restricted within $-\pi / 2<\theta<\pi / 2$, which is justified by the common practice to 


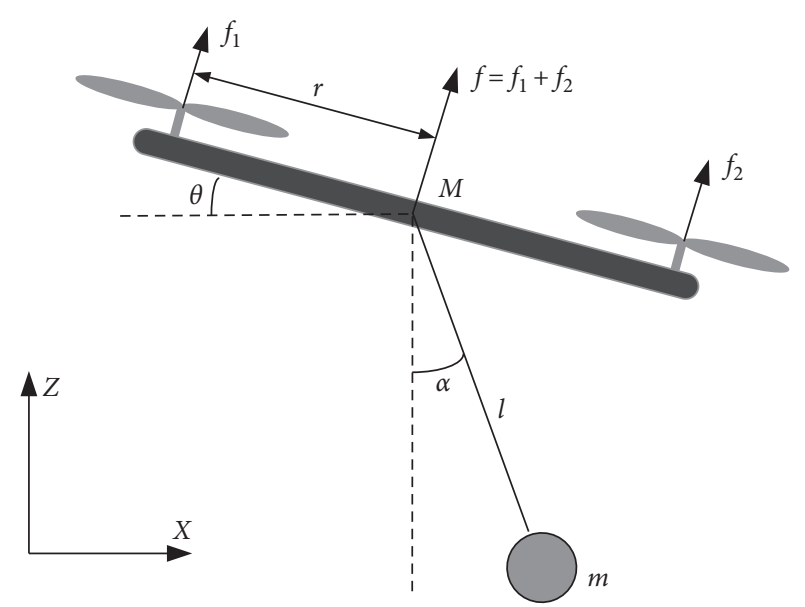

Figure 1: Quadrotor transporting a payload in the $X-Z$ plane.

limit the quadrotor capability for longitudinal/lateral motion in hover flight, and decomposing the total thrust to the $f_{x}=f \sin \theta$ and $f_{z}=f \cos \theta$ components acting the quadrotor motion along the $x$ - and $z$-axis, respectively, consider the redefinition of the model (4) as follows:

$$
\begin{aligned}
& \ddot{x}=\frac{M+m \cos ^{2} \alpha}{M(M+m)} f_{x}+\frac{m \sin \alpha \cos \alpha}{M(M+m)} f_{z}+\frac{m l \dot{\alpha}^{2} \sin \alpha}{M+m}, \\
& \ddot{z}=\frac{m \sin \alpha \cos \alpha}{M(M+m)} f_{x}+\frac{M+m \sin ^{2} \alpha}{M(M+m)} f_{z}-\frac{m l \dot{\alpha}^{2} \cos \alpha}{M+m}-g, \\
& \ddot{\alpha}=-\frac{\cos \alpha}{M l} f_{x}-\frac{\sin \alpha}{M l} f_{z}, \\
& \ddot{\theta}=\frac{\tau}{J_{\theta}}
\end{aligned}
$$

\section{Control Scheme Design}

The control objective is to move the quadrotor from the initial position $\left(x_{0}, z_{0}\right)$ to the desired position $\left(x_{d}, z_{d}\right)$ with suppressing the pendulum oscillation. The control scheme presented in Figure 2 is split into the SMC applied to quadrotor-pendulum horizontal position control, and two feedback linearizing controllers are used for altitude and attitude control. The virtual control signals $f_{x}$ and $f_{z}$, which are the products of sliding mode controller and altitude controller, respectively, are transitioned to the total thrust $f$, as well as the desired pitch angle $\theta_{d}$ is utilized for rotational control of the quadrotor. The virtual force $f_{x}$ is calculated based on $f_{z}$ and, contrariwise, the vertical component of the thrust is necessary to determine $f_{x}$. This creates a loop, in which knowledge of one of the control forces is required beforehand. Thus, in practice, the altitude and horizontal controllers should be performed sequentially to ensure feasibility.

The combination feedback linearization and sliding mode control has been utilized and proven effective in
[20,21] by both obtaining superior performance as opposed to classical PI controllers but also in being able to stabilize the system at varying operating conditions. Sliding mode controllers suffer from the chattering phenomenon which is most commonly encountered during practical implementation. The phenomenon is caused by unmodelled dynamics such as those from sensors, data processors, etc. Several methods of mitigating chattering can be found in the literature which includes using higher-order sliding modes (HOSM) [22], boundary layer [23], and observer-based chattering suppression [24]. To alleviate the problem of chattering in the quadrotor-pendulum system, we divide the control input into a continuous equivalent and the discontinuous switching part which reduces the switching amplitude.

3.1. Horizontal Motion Control. Consider the dynamic model of a quadrotor with suspended pendulum moving along the $x$-axis can be described using the state space expression:

$$
\begin{aligned}
& \dot{x}_{1}=x_{2}, \\
& \dot{x}_{2}=g_{1}(x)+b_{1}(x) f_{x}, \\
& \dot{x}_{3}=x_{4}, \\
& \dot{x}_{4}=g_{2}(x)+b_{2}(x) f_{x},
\end{aligned}
$$

where $x=\left[x_{1}, x_{2}, x_{3}, x_{4}\right]^{T}=\left[x-x_{d}, \dot{x}-\dot{x}_{d}, \alpha, \dot{\alpha}\right]^{T}$ and $g_{i}$ and $b_{i}(i=1,2)$ for $f_{z}$ assumed as the time-varying parameter are given by

$$
\begin{aligned}
& g_{1}(x)=\frac{m \sin \alpha \cos \alpha}{M(M+m)} f_{z}+\frac{m l \dot{\alpha}^{2} \sin \alpha}{M+m}-\ddot{x}_{d}, \\
& b_{1}(x)=\frac{M+m \cos ^{2} \alpha}{M(M+m)} \\
& g_{2}(x)=-\frac{\sin \alpha}{M l} f_{z}, \\
& b_{2}(x)=-\frac{\cos \alpha}{M l}
\end{aligned}
$$

The sliding surface $s$ is proposed as

$$
s=c_{1} x_{1}+x_{2}+c_{2} x_{3}+c_{3} x_{4}
$$

where $c_{1}, c_{2}$, and $c_{3}$ are the sliding-mode control parameters.

Splitting the control signal $f_{x}$ into equivalent $u_{\mathrm{eq}}$ and switching control $u_{\text {sw }}$,

$$
f_{x}=u_{\mathrm{eq}}+u_{\mathrm{sw}}
$$

The equivalent control is derived from $\dot{s}=0$ :

$$
\begin{aligned}
\dot{s} & =c_{1} x_{2}+\dot{x}_{2}+c_{2} x_{4}+c_{3} \dot{x}_{4} \\
& =c_{1} x_{2}+g_{1}(x)+b_{1}(x) f_{x}+c_{2} x_{4}+c_{3}\left(g_{2}(x)+b_{2}(x) f_{x}\right),
\end{aligned}
$$

which through defining $f_{x}=u_{\text {eq }}$ results in

$$
u_{\mathrm{eq}}=-\frac{c_{1} x_{2}+c_{2} x_{4}+g_{1}(x)+c_{3} g_{2}(x)}{b_{1}(x)+c_{3} b_{2}(x)} \text {. }
$$




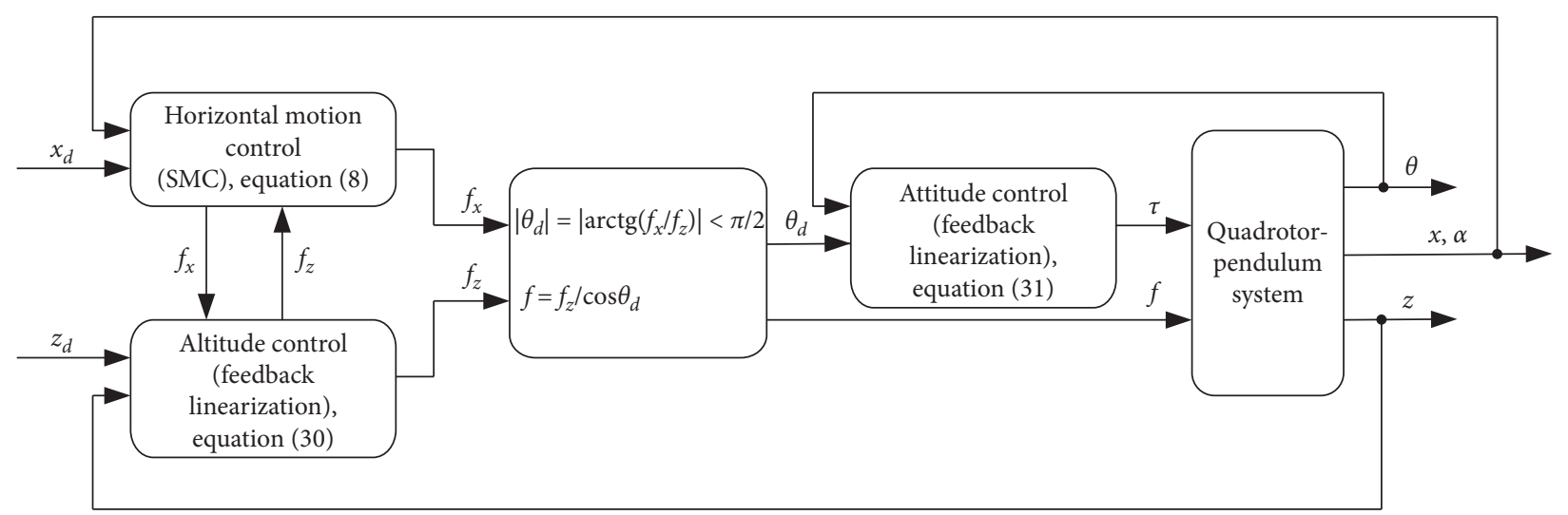

Figure 2: Structure of the control system.

The sliding-mode control parameters can be determined through assigning the eigenvalues of the linearized closedloop system (6) at specified location. Hence, linearizing the closed-loop system at an equilibrium point $x=[0,0,0,0]^{T}$, the closed-loop (6) can be expressed as

$$
\begin{aligned}
\dot{x}_{2} & =g_{1}(x)+b_{1}(x) u_{\mathrm{eq}} \\
& =\frac{c_{3} f_{z}}{(M+m)\left(l-c_{3}\right)} x_{3}-\frac{l}{l-c_{3}}\left(c_{1} x_{2}+c_{2} x_{4}\right), \\
\dot{x}_{4} & =g_{2}(x)+b_{2}(x) u_{\mathrm{eq}} \\
& =-\frac{f_{z}}{(M+m)\left(l-c_{3}\right)} x_{3}+\frac{1}{l-c_{3}}\left(c_{1} x_{2}+c_{2} x_{4}\right),
\end{aligned}
$$

or in the simplified compact form $\dot{x}=A x$ :

$$
\left[\begin{array}{c}
\dot{x}_{2} \\
\dot{x}_{3} \\
\dot{x}_{4}
\end{array}\right]=\left[\begin{array}{ccc}
-\frac{c_{1} l}{l-c_{3}} & \frac{c_{3} f_{z}}{(M+m)\left(l-c_{3}\right)} & -\frac{c_{2} l}{l-c_{3}} \\
0 & 0 & 1 \\
\frac{c_{1}}{l-c_{3}} & \frac{f_{z}}{(M+m)\left(l-c_{3}\right)} & \frac{c_{2}}{l-c_{3}}
\end{array}\right]\left[\begin{array}{c}
x_{2} \\
x_{3} \\
x_{4}
\end{array}\right] .
$$

The parameters $c_{1}, c_{2}$, and $c_{3}$ should be selected such that $A$ is Hurwitz. Since the characteristic polynomial is given by

$$
\begin{aligned}
\operatorname{det}(s I-A)= & s^{3}+\frac{c_{1} l-c_{2}}{l-c_{3}} s^{2}+\frac{f_{z}}{(M+m)\left(l-c_{3}\right)} s \\
& +\frac{c_{1} f_{z}}{(M+m)\left(l-c_{3}\right)}
\end{aligned}
$$

for the desired Hurwitz polynomial

$$
P(s)=s^{3}+p_{2} s^{2}+p_{1} s+p_{0},
$$

the parameters of sliding-mode controller should be

$$
\begin{aligned}
& c_{1}=\frac{p_{0}}{p_{1}}, \\
& c_{2}=c_{1} l-\frac{p_{2}}{p_{1}(M+m)} f_{z}, \\
& c_{3}=l-\frac{f_{z}}{p_{1}(M+m)},
\end{aligned}
$$

where $f_{z}$ is considered as the time-varying parameter. Since the quadrotor's pitch angle is restricted within $-\pi / 2<$ $\theta<\pi / 2$, the system will be asymptotically stable as long as $f_{z}>0$.

Consider the Lyapunov candidate function in the form

$$
V=\frac{1}{2} s^{2}
$$

Differentiating (12) with respect to time and substituting (6) and (8)-(10), we have

$$
\begin{aligned}
\dot{V}= & s \dot{s}=s\left[c_{1} \dot{x}_{1}+c_{2} \dot{x}_{3}+g_{1}(x)+c_{3} g_{2}(x)\right. \\
& \left.+\left(b_{1}(x)+c_{3} b_{2}(x)\right)\left(u_{\mathrm{eq}}+u_{\mathrm{sw}}\right)\right] \\
= & s\left(b_{1}(x)+c_{3} b_{2}(x)\right) u_{\mathrm{sw}} .
\end{aligned}
$$

Choosing the switching control as

$$
u_{\mathrm{sw}}=-\frac{\eta_{1} s+\eta_{2} \operatorname{sgn}(s)}{b_{1}(x)+c_{3} b_{2}(x)},
$$

leads to

$$
\dot{V}=-\eta_{1} s^{2}-\eta_{2}|s| \leq 0
$$

where $\eta_{1}$ and $\eta_{2}$ are the positive constants.

Equation (20) will be locally negative definite (except the trivial solution $x_{1}=x_{2}=x_{3}=x_{4}=0$ ) in the neighborhood of the equilibrium point taking into account some limitations of the sliding surface parameters. For $s=0$, we have

$$
c_{3}=-\frac{c_{1} x_{1}+x_{2}+c_{2} x_{3}}{x_{4}} .
$$

To find the limit value of $c_{3}$, consider system (6) converges to the equilibrium $x=0$ as $t \longrightarrow \infty$. Applying de L'Hospital rule in (22), the limit value of $c_{3}$ is determined as 


$$
\begin{aligned}
c_{3} & <\lim _{x \longrightarrow 0}-\frac{c_{1} x_{1}+x_{2}+c_{2} x_{3}}{x_{4}} \stackrel{H}{=} \lim _{x \longrightarrow 0}-\frac{c_{1} x_{2}+\dot{x}_{2}+c_{2} x_{4}}{\dot{x}_{4}} \\
& =\lim _{x \longrightarrow 0}-\frac{c_{1} x_{2}+c_{2} x_{4}+g_{1}(x)+b_{1}(x) f_{x}}{g_{2}(x)+b_{2}(x) f_{x}}=l .
\end{aligned}
$$

Proceeding in the same manner for $c_{2}$, the limits of sliding surface parameters are found as follows:

$$
\begin{aligned}
& c_{2}<c_{1} l, \\
& c_{3}<l .
\end{aligned}
$$

Please note that the same conditions as (23) can be also directly established from (14). Thus, the limit values of $c_{2}$ and $c_{3}$ correspond to the singularity of the closed-loop matrix $A(x)$ (13). Substituting (16) into (23), it is enough to state that the matrix $A(x)$ is nonsingular everywhere as long as $f_{z}=f \cos \theta>0 \Longrightarrow|\theta|<\pi / 2$.

Integrating both sides of (20), we obtain

$$
V(t)-V(0)=\int_{0}^{t}\left(-\eta_{1} s^{2}-\eta_{2}|s|\right) \mathrm{d} t
$$

Furthermore, we have

$$
V(0)=V(t)+\int_{0}^{t}\left(\eta_{1} s^{2}+\eta_{2}|s|\right) \mathrm{d} t \geq \int_{0}^{t}\left(\eta_{1} s^{2}+\eta_{2}|s|\right) \mathrm{d} t .
$$

For the closed-loop satisfying (23) $\left(c_{2}, c_{3}\right.$, and $\theta$ are bounded), from (24), we have

$$
V(t)=V(0)-\int_{0}^{t}\left(\eta_{1} s^{2}+\eta_{2}|s|\right) \mathrm{d} t<V(0)<\infty .
$$

Thus,

$$
\lim _{t \rightarrow \infty} \int_{0}^{t}\left(\eta_{1} s^{2}+\eta_{2}|s|\right) \mathrm{d} t<V(0)<\infty,
$$

and there exist

$$
\begin{aligned}
& \lim _{t \rightarrow \infty} \int_{0}^{t}\left(\eta_{1} s^{2}\right) \mathrm{d} t<\infty, \\
& \lim _{t \rightarrow \infty} \int_{0}^{t}\left(\eta_{2}|s|\right) \mathrm{d} t<\infty .
\end{aligned}
$$

Applying Barbalat's lemma, we can conclude that

$$
\lim _{t \longrightarrow \infty} s=0 \text {. }
$$

If any state diverges, such that $\lim _{t \rightarrow \infty} x_{i}=\infty(i=1,2$, 3 , 4) and $\lim _{t \rightarrow \infty} s=\infty$, which contradict (29), it implies that as long as $f_{z}=f \cos \theta>0 \Longrightarrow|\theta|<\pi / 2$, the closed-loop matrix is nonsingular and all states asymptotically converge to equilibrium point.

3.2. Altitude and Attitude Control. The feedback linearization procedure is applied to develop the altitude and attitude control of a quadrotor. The quadrotor's vertical and rotational motion is described by

$$
\begin{aligned}
& \ddot{z}=\frac{m \sin \alpha \cos \alpha}{M(M+m)} f_{x}+\frac{M+m \sin ^{2} \alpha}{M(M+m)} f_{z}-\frac{m l \dot{\alpha}^{2} \cos \alpha}{M+m}-g, \\
& \ddot{\theta}=\frac{\tau}{J_{\theta}},
\end{aligned}
$$

where $f_{x}$ is assumed as the time-varying parameter.

Choosing the control inputs as

$$
\begin{aligned}
f_{z} & =\frac{M(M+m)\left(-k_{p z} e_{z}-k_{d z} \dot{e}_{z}+\ddot{z}_{d}+g\right)+m M l \dot{\alpha}^{2} \cos \alpha-f_{x} m \sin \alpha \cos \alpha}{M+m \sin ^{2} \alpha} \\
\tau & =-J_{\theta}\left(k_{p \theta} e_{\theta}+k_{d \theta} \dot{e}_{\theta}-\ddot{\theta}_{d}\right)
\end{aligned}
$$

where $e_{z}=z-z_{d}$ and $e_{\theta}=\theta-\theta_{d}$ are tracking errors, $k_{p z}$, $k_{d z}, k_{p \theta}$, and $k_{d \theta}$ are the positive control parameters, and $\theta_{d}$ is the desired pitch angle:

$$
\left|\theta_{d}\right|=\left|\operatorname{arctg}\left(\frac{f_{x}}{f_{z}}\right)\right|<\frac{\pi}{2}
$$

\section{Simulation Results}

There are three groups of simulation results analyzed in this section to verify effectiveness of the proposed control approach developed for the quadrotor transporting suspended payload. In the first group, we investigate the control system performances for the step input function with respect to the variation of the pendulum length and we test the tracking performances. The second group of numerical simulations compares the control method proposed in this paper with the partial feedback linearizing controller (PFLC) and feedback linearizing controller combined with input shaping. The last simulation group was performed to analyze the robustness of the proposed method under parameter uncertainty.

4.1. Performances with Different Operating Conditions. The numerical simulations were carried out for different rope lengths 1,2 , and $4 \mathrm{~m}$, respectively, and constant mass of the payload $m=0.4 \mathrm{~m}$. The quadrotor parameters are presented in Table 1, together with parameters of the altitude and attitude controllers which were empirically chosen.

The closed-loop eigenvalues were located at $\lambda=-\omega=-\sqrt{g / l}$, where $\omega$ is the natural undamped 
TABle 1: Parameters of the model and altitude, and attitude controllers.

\begin{tabular}{cccccccc}
\hline & \multicolumn{2}{c}{$\begin{array}{c}\text { Model } \\
\text { parameters }\end{array}$} & & \multicolumn{2}{c}{$\begin{array}{c}\text { Altitude } \\
\text { control }\end{array}$} & \multicolumn{2}{c}{$\begin{array}{c}\text { Attitude } \\
\text { control }\end{array}$} \\
$M(\mathrm{~kg})$ & $J_{\theta}\left(\mathrm{kgm}^{2}\right)$ & $m(\mathrm{~kg})$ & $g\left(\mathrm{~m} / \mathrm{s}^{2}\right)$ & $k_{p z}$ & $k_{d z}$ & $k_{p \theta}$ & $k_{d \theta}$ \\
\hline 2 & 0.17 & 0.4 & 9.81 & 4 & 4 & 200 & 20 \\
\hline
\end{tabular}

frequency of a pendulum, which results in the SMC performing with sliding surface parameters determined according to (16):

$$
\begin{aligned}
& c_{1}=\frac{\omega}{3}, \\
& c_{2}=\frac{l \omega}{3}-\frac{f_{z}}{\omega(M+m)}, \\
& c_{3}=l-\frac{f_{z}}{3 \omega^{2}(M+m)} .
\end{aligned}
$$

Table 2 presents the sliding surface parameter $c_{1}$ and the limit values of $c_{2}$ and $c_{3}$.

The rest of the SMC parameters were set as $\eta_{1}=1$ and $\eta_{2}=0.01$. Figures 3-6 present the system unit-step input responses, while the settling time of quadrotor position (measured with tolerance margin of $2 \%$ ) and maximum absolute value of the swing angle are presented in Table 3. The simulations proved that the pendulum vibration is effectively suppressed for different operating conditions. The horizontal desired position is achieved without overshoot, as the eigenvalues of the closed-loop are located at $\lambda=-\omega$, within 5, 5.6, and 6.9 seconds, while the maximum amplitude of the pendulum swing angle is 0.061, 0.04, and
$0.026 \mathrm{rad}$ for rope lengths 1,2 , and 4 meters, respectively. The response of the altitude subsystem also reaches the desired position $z_{d}$ without overshoot within $3 \mathrm{~s}$. For the assumed strategy of assigning the closed-loop eigenvalues $\lambda=-\omega$, the settling time and pendulum swing angle amplitude do not depend on the mass of the payload and only the rope length influences these performance indices. The specific performance criteria can be met by choosing proper location of closed-loop poles and tuning the altitude and attitude control parameters.

Figure 7 presents the quadrotor position in the $X-Z$ plane and pendulum swing angle when the quadrotor with cablesuspended payload follows the trajectory given by

$$
\left[\begin{array}{l}
x_{d}(t) \\
z_{d}(t)
\end{array}\right]=\left[\begin{array}{c}
\cos t+0.3 t-1 \\
\cos t+t-1
\end{array}\right] \text {. }
$$

The simulation, which was carried out for $m=0.5 \mathrm{~kg}$ and $l=0.5 \mathrm{~m}$, demonstrates good tracking performances with maximum error $0.06 \mathrm{~m}$ respect to the reference trajectory, while the amplitude of the pendulum swing is mitigated within $0.126 \mathrm{rad}$.

4.2. Comparison with PFLC and ZVDD. In this group of simulations, the control scheme described in this paper is compared with the open- and closed-loop techniques used for reducing the pendulum oscillation. As the first example, the feedback linearizing controller combined with input shaping is proposed and developed. In this control strategy, the altitude and attitude control laws (31) and (32), respectively, are completed by the horizontal position controller given as

$$
f_{x}=\frac{M(M+m)\left(-k_{p x} e_{x}-k_{d x} \dot{e}_{x}+\ddot{x}_{d}\right)-m M l \dot{\alpha}^{2} \sin \alpha-f_{z} m \sin \alpha \cos \alpha}{M+m \cos ^{2} \alpha},
$$

where $e_{x}=x-x_{d}$, and $k_{p x}$ and $k_{d x}$ are the positive parameters. Thus, the feedback control laws (31), (32), and (36) are adapted for positioning the quadrotor in the $X-Z$ plane, while the feedforward input shaping filter is incorporated to reduce pendulum oscillations. A ZVDD input shaper was chosen, as this shaper provides more robustness against variation of the system's natural frequency compared to the ZV and ZVD shapers [9]. The general formula for the amplitudes $A_{i}$ and time locations $t_{i}$ of the impulse sequence is as follows:

$$
\left[\begin{array}{c}
A_{i} \\
t_{i}
\end{array}\right]=\left[\begin{array}{cccc}
\frac{1}{(K+1)^{3}} & \frac{3 K}{(K+1)^{3}} & \frac{3 K^{2}}{(K+1)^{3}} & \frac{3 K^{3}}{(K+1)^{3}} \\
0 & \frac{\pi}{\omega_{d}} & \frac{2 \pi}{\omega_{d}} & \frac{3 \pi}{\omega_{d}}
\end{array}\right],
$$

where $\quad K=\exp \left(-\xi \pi / \sqrt{1-\xi^{2}}\right), \quad \omega_{d}=\omega \sqrt{1-\xi^{2}}, \quad$ and $\sum_{i=1}^{4} A_{i}=1$.

The series of impulses (37) were convolved with input signal $f_{x}(36)$. The amplitudes and their time locations (37) were determined for natural undamped frequency of the pendulum $\omega$ and by estimating the damping ratio as $\zeta=0.273$. The parameters of the feedback controller (36) were set to $k_{p x}=1$ and $k_{d x}=2$, while the rest of the parameters were set as in the previous simulations.

As the second comparative control approach, the PFLC proposed in [25] is applied for quadrotor positioning in the horizontal direction and suppressing the payload oscillation with the control law:

$$
f_{x}=-k_{p} e_{x}-k_{d} \dot{e}_{x}+\frac{k_{\alpha} l}{\cos \alpha} \dot{\alpha}
$$

where the controller parameters were chosen as $k_{p}=1$, $k_{d}=2.65$, and $k_{\alpha}=10$. 
TABLE 2: $c_{1}$ and limits of $c_{2}$ and $c_{3}$.

\begin{tabular}{lccc}
\hline Rope length & $1 \mathrm{~m}$ & $2 \mathrm{~m}$ & $4 \mathrm{~m}$ \\
\hline$c_{1}$ & 1.0440 & 0.7382 & 0.5220 \\
Limit of $c_{2}$ & 1.0440 & 1.4765 & 2.0881 \\
Limit of $c_{3}$ & 1 & 2 & 4 \\
\hline
\end{tabular}

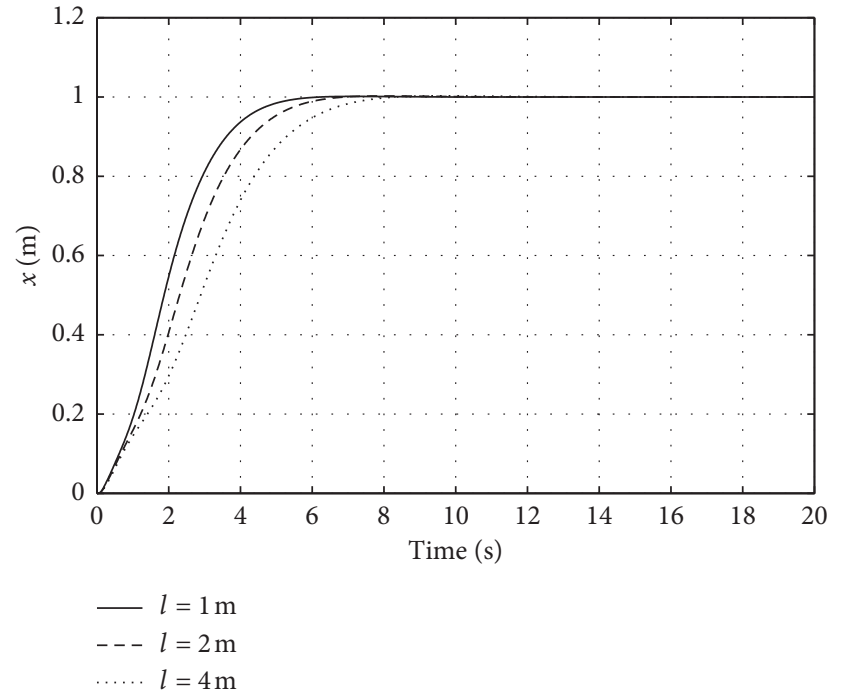

(a)

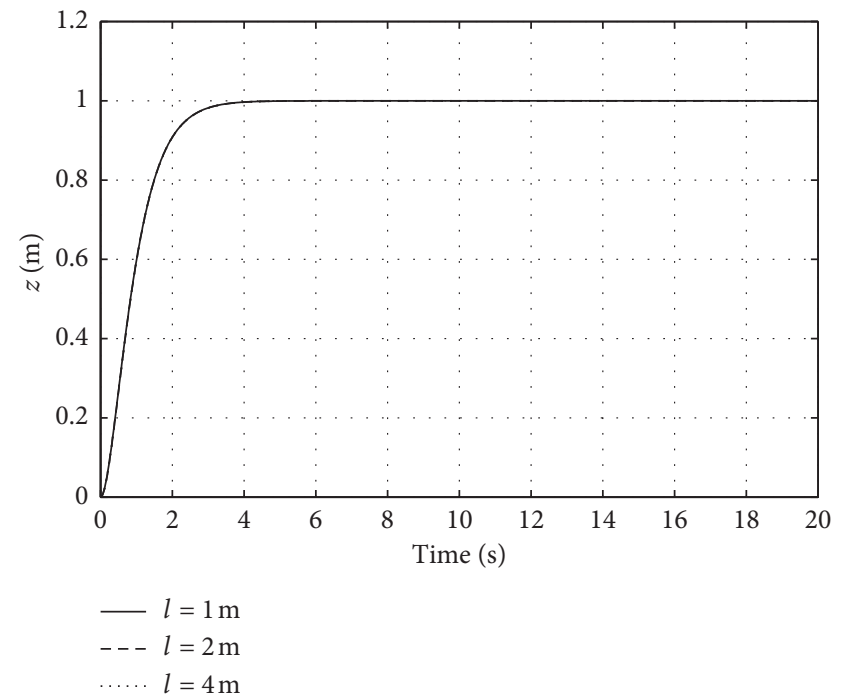

(b)

FIgURE 3: Quadrotor positions along the $x$ - and $z$-axis.

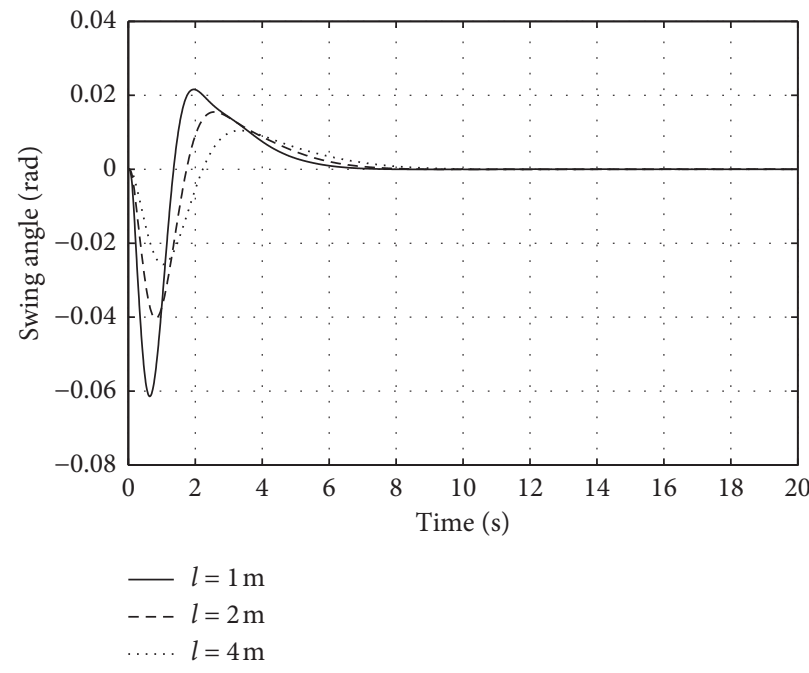

(a)

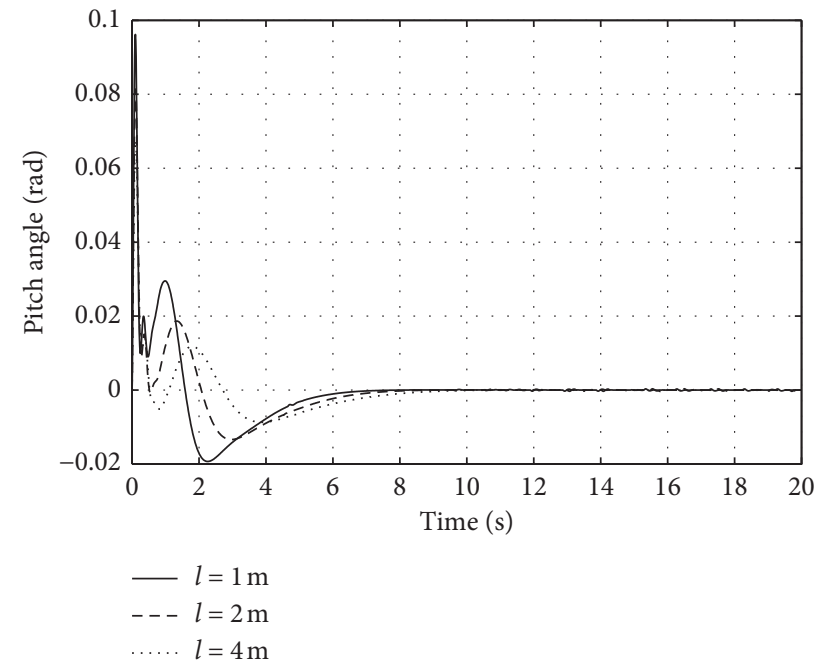

(b)

Figure 4: Swing and pitch angle.

In this simulation scenario, the SMC performs with the sliding surface parameters (16) determined for the closedloop eigenvalues located at $\lambda=-0.5 \omega$, while the parameters of the altitude and attitude controllers are the same as in the previous simulations.

The simulations were carried out for $m=0.5 \mathrm{~kg}, l=0.5 \mathrm{~m}$, and $x_{d}=z_{d}=1 \mathrm{~m}$. Figure 8 presents the step responses and thrust component $f_{x}$ of three control strategies, denoted SMC, PFLC, and ZVDD, while Table 4 presents the settling time of quadrotor's horizontal position (measured with tolerance margin of $2 \%$ ) and maximum absolute value of the swing angle. The settling time is similar, $6.2 \mathrm{~s}, 6.3 \mathrm{~s}$, and $6.4 \mathrm{~s}$, for the SMC, PFL, and ZVDD, respectively, which was achieved by assigning the SMC eigenvalues at $\lambda=-0.5 \omega$. 


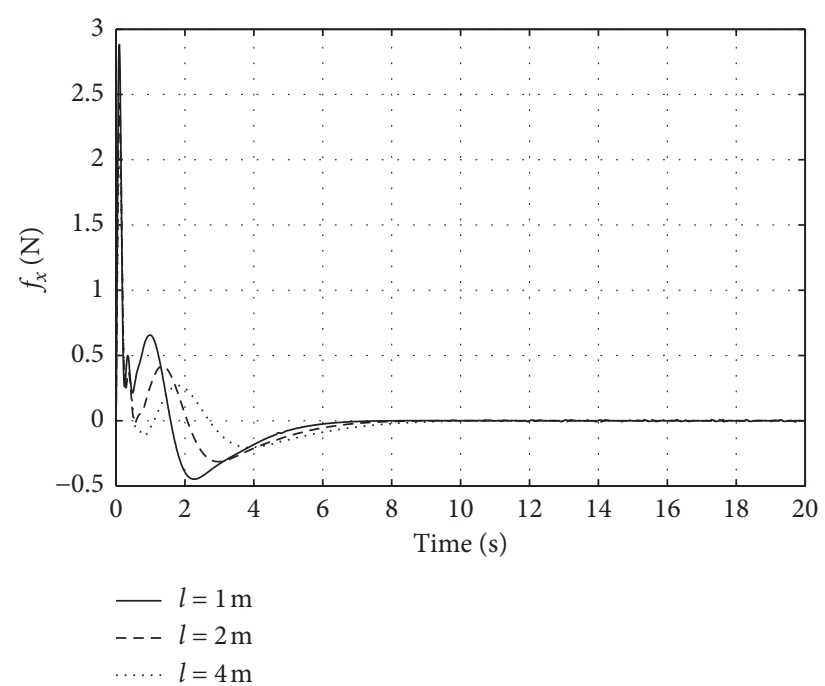

(a)

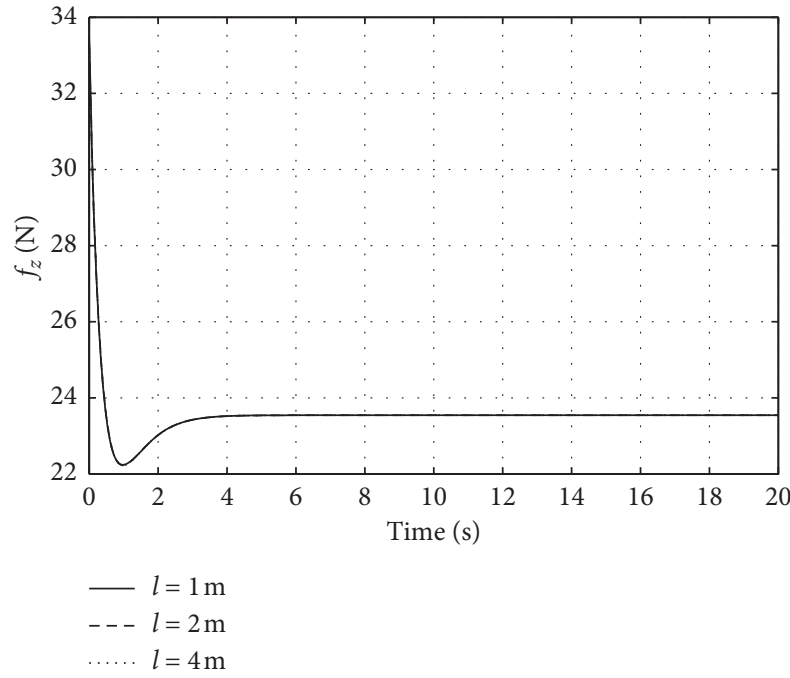

(b)

Figure 5: Thrust components $f_{x}$ and $f_{z}$.

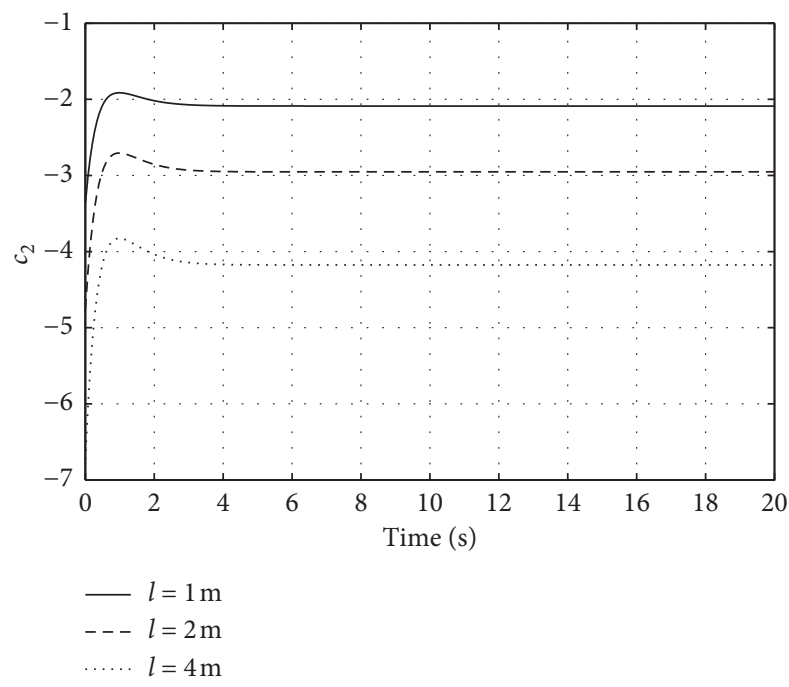

(a)

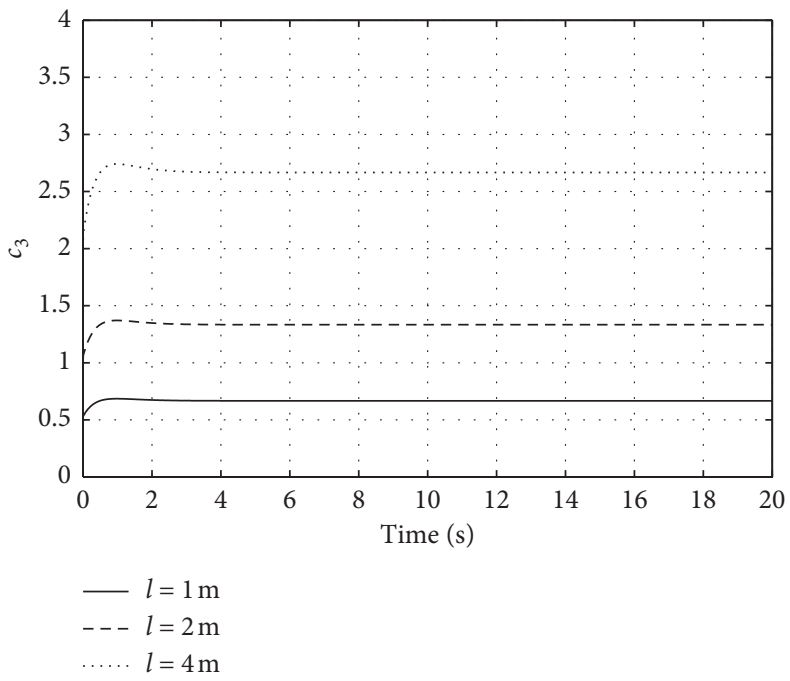

(b)

Figure 6: Sliding surface parameter $c_{2}$ and $c_{3}$.

TABle 3: Performances of the proposed control scheme for different operating conditions.

\begin{tabular}{lccc}
\hline Rope length & $1 \mathrm{~m}$ & $2 \mathrm{~m}$ & $4 \mathrm{~m}$ \\
\hline Settling time in $x$ direction & $5 \mathrm{~s}$ & $5.6 \mathrm{~s}$ & $6.9 \mathrm{~s}$ \\
Settling time in $z$ direction & $3 \mathrm{~s}$ & $3 \mathrm{~s}$ & $3 \mathrm{~s}$ \\
Maximum swing angle & $0.061 \mathrm{rad}$ & $0.04 \mathrm{rad}$ & $0.026 \mathrm{rad}$ \\
\hline
\end{tabular}

Thus, there is minor difference in reduction of pendulum oscillation in terms of the time, but the SMC better suppresses the pendulum oscillation in the transient state. The maximum amplitude of the pendulum swing angle is $0.030 \mathrm{rad}, 0.035 \mathrm{rad}$, and $0.047 \mathrm{rad}$ for the SMC, PFLC, and ZVDD, respectively (over $56 \%$ better payload swing reduction comparing to the ZVDD). In case of the ZVDD, the small residual vibration is produced, less than $0.0007 \mathrm{rad}$, which is not observed in case of the SMC and PFLC. The PFLC performs similarly to the SMC; however, the performances of the PFLC deteriorate when subject to operating conditions. Figure 9 presents comparison of the step responses for the SMC and PFLC for $l=2 \mathrm{~m}$ and $m=0.5 \mathrm{~kg}$. The PFLC exhibits significant deterioration of performances in the presence of changing the operating conditions. The overshoot, settling time, and maximum payload swing angle are $7 \%, 12.7 \mathrm{~s}$, and $0.0234 \mathrm{rad}$, respectively, for PFLC, while the SMC performing with the parameters (16) determined for closed-loop eigenvalues located at $\lambda=-0.5 \omega$ results in settling time and maximum swing angle of $9.9 \mathrm{~s}$ and $0.0115 \mathrm{rad}$, respectively. The simulation results confirm the 


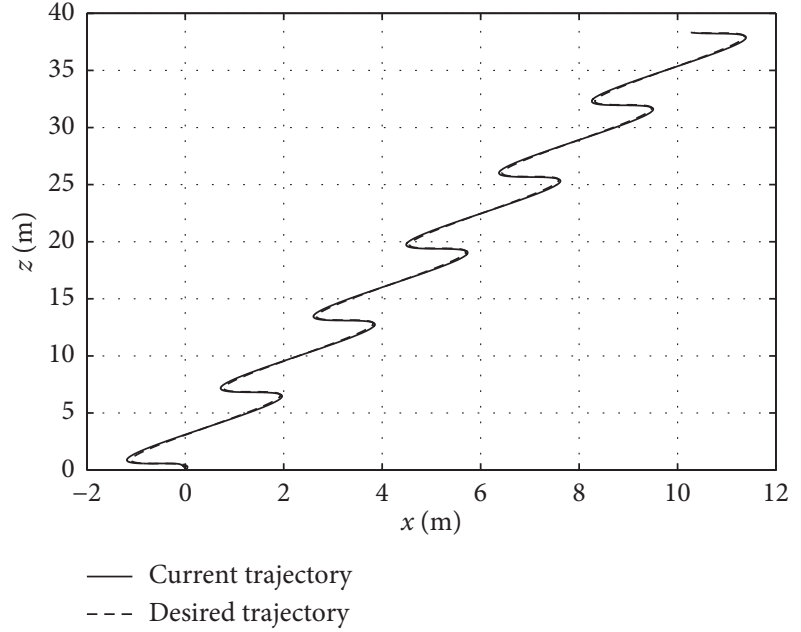

(a)

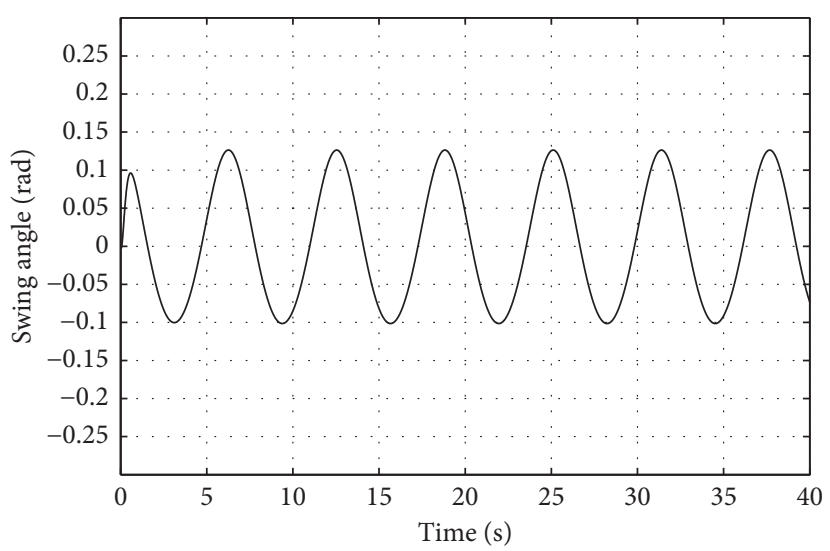

(b)

Figure 7: Trajectory of the quadrotor in $X-Z$ plane and pendulum swing angle.
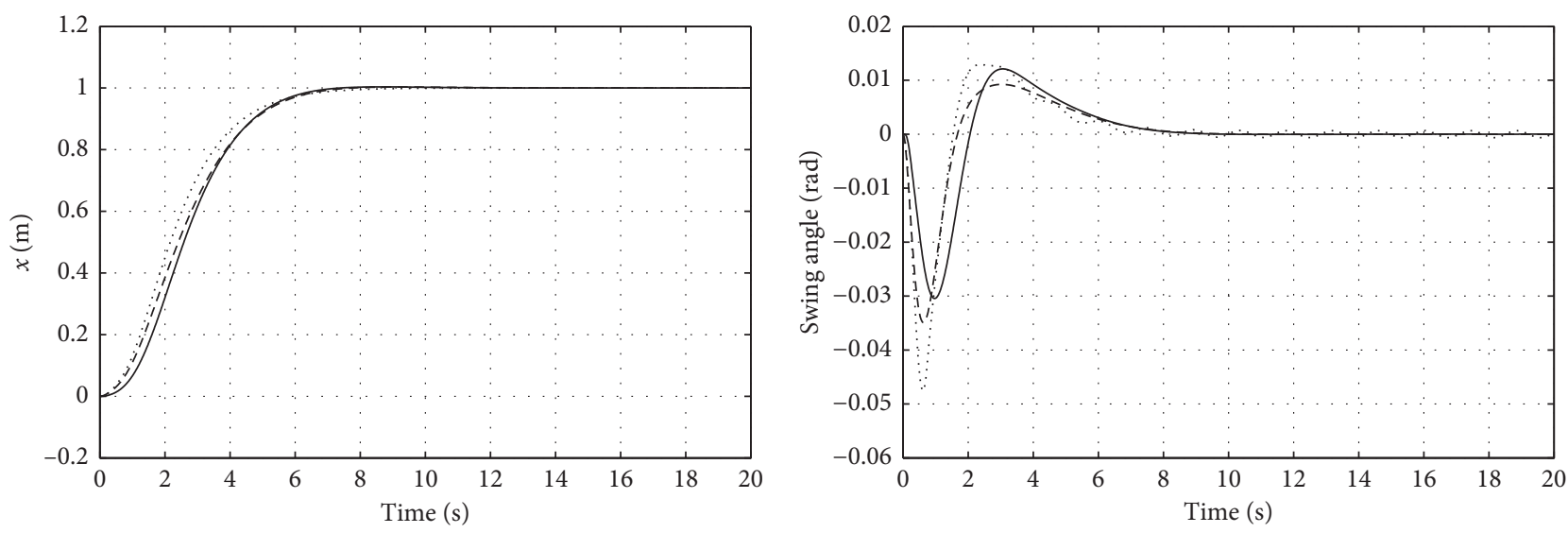

- SMC
--- PFLC
$\ldots .$. ZVDD

- SMC

- - PFLC

ZVDD

(a)

(b)

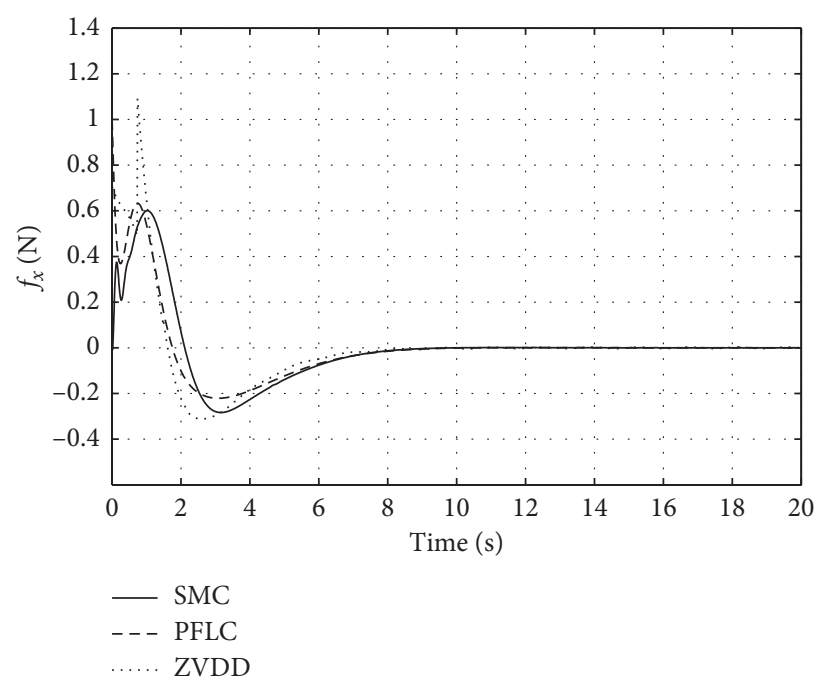

(c)

FIGURE 8: Quadrotor's position along the $X$-axis, pendulum swing angle, and force $f_{x}$, comparison of the proposed SMC with PFLC and ZVDD controllers for $l=0.5 \mathrm{~m}$ and $m=0.5 \mathrm{~kg}$. 
TABLE 4: Performances of SMC, PFLC, and ZVDD controllers (simulation for $l=0.5 \mathrm{~m}$ and $m=0.5 \mathrm{~kg}$ ).

\begin{tabular}{lccc}
\hline Controller & SMC & PFLC & ZVDD \\
\hline Settling time in $x$ direction & $6.2 \mathrm{~s}$ & $6.3 \mathrm{~s}$ & $6.4 \mathrm{~s}$ \\
Maximum swing angle & $0.030 \mathrm{rad}$ & $0.035 \mathrm{rad}$ & $0.047 \mathrm{rad}$ \\
\hline
\end{tabular}

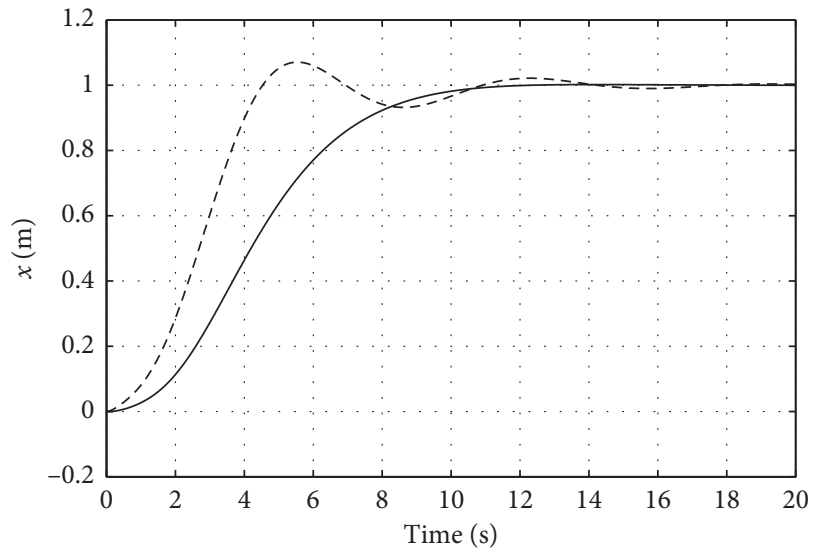

- SMC

-. - PFLC

(a)

(a)

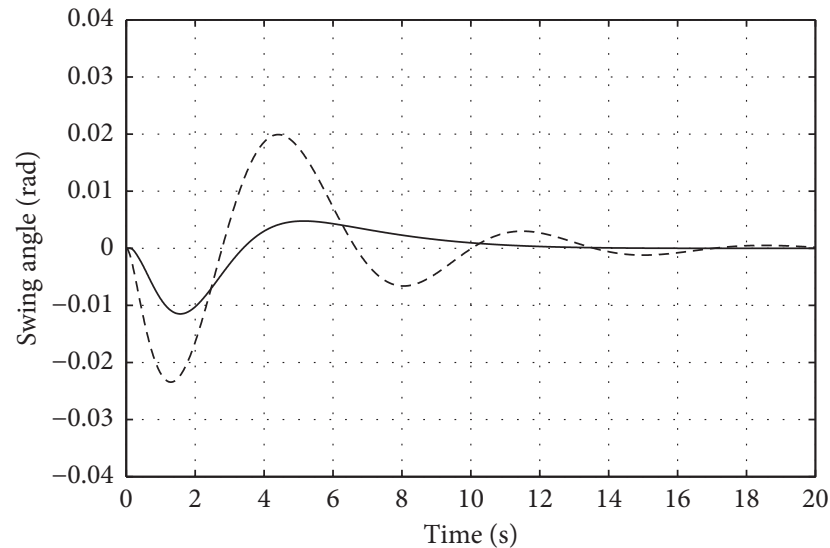

- SMC

- . - PFLC

(b)

FIgURE 9: Quadrotor's position along the $X$-axis and pendulum swing angle, comparison of the proposed SMC with the PFLC controller for $l=2 \mathrm{~m}$ and $m=0.5 \mathrm{~kg}$.

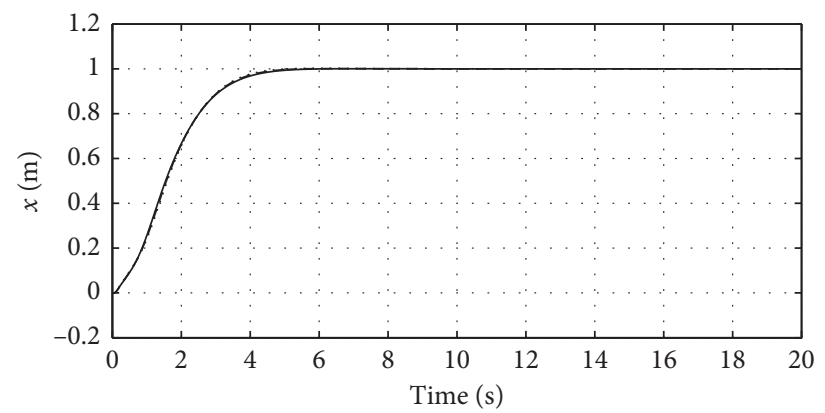

$-l=0.5 \mathrm{~m}, \mathrm{~m}=0.5 \mathrm{~m}$

- - - Uncertainty range of $20 \%$

..... Uncertainty range of $40 \%$

(a)

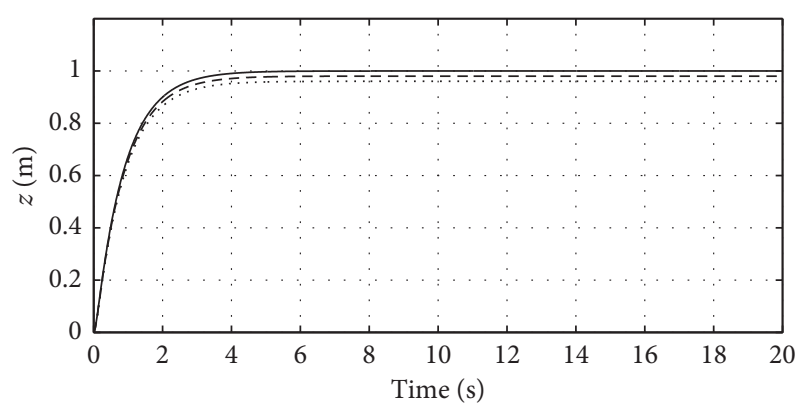

$l=0.5 \mathrm{~m}, m=0.5 \mathrm{~kg}$

- - - Uncertainty range of $20 \%$

Uncertainty range of $40 \%$

(b)

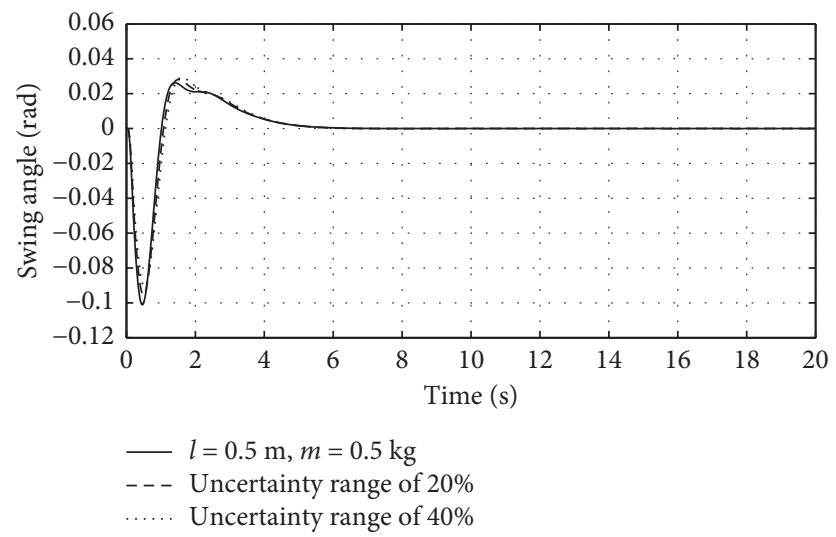

(c)

Figure 10: Quadrotor positions along the $X$ - and $Z$-axis, and pendulum swing angle-responses for $l=0.5 \mathrm{~m}$ and $m=0.5 \mathrm{~kg}$, and for parameter perturbation with the range of $20 \%$ and $40 \%$. 
effectiveness of the proposed control approach for positioning the quadrotor with cable-suspended payload and suppressing the transient and residual pendulum oscillations.

4.3. Parameter Uncertainty. To evaluate the performance of the proposed controller under parameter uncertainty, simulations were carried out for perturbation of rope length and payload mass within the ranges of $20 \%$ and $40 \%$, respectively. Assuming the nominal operating conditions as $l=0.5 \mathrm{~m}$ and $m=0.5 \mathrm{~kg}$, the parameters of the model (5) were increased by $20 \%$ and $40 \%$, whereas the proposed control scheme performed during simulations for nominal values of the rope length and payload mass. The SMC performed with sliding surface parameters (16) determined for nominal operating point by assigning the closed-loop eigenvalues at $\lambda=-\omega$ and parameters of the altitude and attitude controllers remained as in the previous simulations. Figure 10 presents the responses for the nominal values of rope length and payload mass and uncertainty ranges of $20 \%$ and $40 \%$. The performance of the SMC applied for positioning the quadrotor in $x$ direction and suppressing the pendulum oscillation is not much affected by the parameter uncertainty: settling time remains within 4.1 and $4.3 \mathrm{~s}$, while the maximum swing angle is within 0.09 and $0.1 \mathrm{rad}$. In case of the altitude feedback linearization controller, the small steady-state error is observed $(0.02 \mathrm{~m}$ and $0.04 \mathrm{~m}$ for the parameters increase by $20 \%$ and $40 \%$, respectively). Thus, in case of the plant and model mismatch, the deviation between the output $(z)$ and its setpoint can be eliminated by incorporating the integral action to the altitude control law (31).

\section{Conclusions}

In this paper, the positioning and damping of transient and residual vibrations of a quadrotor with a cable-suspended payload is considered. The coupled quadrotor-pendulum dynamics were derived using the Euler-Lagrange formulation in the $X-Z$ plane. The attitude and altitude dynamics were controlled using a feedback linearizing controller. To reduce payload vibration caused by horizontal motion, a sliding mode controller was used with sliding surface parameters tuned using the adaptive pole placement method with the vertical component of the thrust taken as a time varying parameter. Asymptotic stability of the closed-loop system was proved as long as the adaptive sliding surface parameters were less than the rope length. The eigenvalues of the closed-loop system were chosen to be the natural frequency of the pendulum system, and simulations were carried out for different rope lengths.

A comparative analysis was performed between the proposed SMC and a ZVDD input shaper to reduce the pendulum vibrations. The poles of the SMC were placed to obtain similar settling time to be able to compare the pendulum oscillations. The SMC reduced the maximum sway angle by over $56 \%$ and did not have any residual vibrations unlike the ZVDD input shaper.
During practical implementation, the dynamics of the rotor would have to be taken into consideration. The rotor suffers from nonlinearities such as input dead zone due to friction. Methods of overcoming this include using neural networks as was performed in [26]. The nonnegativity condition given by $f_{z}>0$ is a unidirectional input constraint. Future work would include augmenting the sliding mode controller to avoid violating the constraint such as in [27], taking the rotor dynamics into account as well as expanding the model into $3 \mathrm{D}$ space.

\section{Nomenclature}

$\begin{array}{ll}\alpha: & \text { Pendulum sway angle } \\ \theta: & \text { Quadrotor pitch angle } \\ M: & \text { Quadrotor mass } \\ m: & \text { Payload mass } \\ l: & \text { Cable length } \\ f: & \text { Thrust } \\ J_{\theta}: & \text { Quadrotor moment of inertia } \\ \tau: & \text { Pitching moment } \\ \omega: & \text { Pendulum natural frequency } \\ \zeta: & \text { Estimated pendulum damping ratio } \\ \text { UAV: } & \text { Unmanned aerial vehicle } \\ \text { VTOL: } & \text { Vertical take-off landing } \\ \text { SMC: } & \text { Sliding mode control } \\ \text { PFLC: } & \text { Partial feedback linearizing controller }\end{array}$

ZVDD: Zero vibration derivative-derivative input shaper.

\section{Data Availability}

The data used to support the findings of this study are included within the article.

\section{Conflicts of Interest}

The authors declare that there are no conflicts of interest regarding the publication of this paper.

\section{Acknowledgments}

This work was financially supported by the Polish Ministry of Science and Higher Education from funds for year 2019.

\section{References}

[1] M. Orsag, C. Korpela, P. Oh, and S. Bogdan, Aerial Manipulation, Springer International Publishing, NewYork, NY, USA, 2018.

[2] D. K. D. Villa, A. S. Brandão, and M. Sarcinelli-Filho, "Load transportation using quadrotors: a survey of experimental results," in Proceedings of 2018 International Conference on Unmanned Aircraft Systems (ICUAS), pp. 84-93, IEEE, Dallas, TX, USA, June 2018.

[3] H. B. Khamseh, F. Janabi-Sharifi, and A. Abdessameud, "Aerial manipulation-a literature survey," Robotics and Autonomous Systems, vol. 107, pp. 221-235, 2018.

[4] S. Gupte, P. I. T. Mohandas, and J. M. Conrad, "A survey of quadrotor unmanned aerial vehicles," in Proceedings of IEEE Southeastcon, March 2012. 
[5] F. Ruggiero, V. Lippiello, and A. Ollero, "Aerial manipulation: a literature review," IEEE Robotics and Automation Letters, vol. 3, no. 3, pp. 1957-1964, 2018.

[6] P. Foehn, D. Falanga, N. Kuppuswamy, R. Tedrake, and D. Scaramuzza, "Fast trajectory optimization for agile quadrotor maneuvers with a cable-suspended payload," in Proceedings of the 2017 Robotics: Science and Systems, Cambridge, MA, USA, July 2017.

[7] P. J. Cruz, M. Oishi, and R. Fierro, "Lift of a cable suspended load by a quadrotor: a hybrid system approach," in Proceedings of IEEE American Control Conference (ACC), pp. 1887-1892, IEEE, Chicago, IL, USA, July 2015.

[8] W. Singhose, "Command shaping for flexible systems: a review of the first 50 years," International Journal of Precision Engineering and Manufacturing, vol. 10, no. 4, pp. 153-168, 2009.

[9] J. Vaughan, A. Yano, and W. Singhose, "Comparison of robust input shapers," Journal of Sound and Vibration, vol. 315, no. 4-5, pp. 797-815, 2008.

[10] J. Huang, X. Xie, and Z. Liang, "Control of bridge cranes with distributed mass payload dynamics," IEEE/ASME Transactions on Mechatronics, vol. 20, no. 1, pp. 481-486, 2015.

[11] S. Sadr, S. A. A. Moosavian, and P. Zarafshan, "Dynamics modeling and control of a quadrotor with swing load," Journal of Robotics, vol. 2014, Article ID 265897, 12 pages, 2014.

[12] N. S. Zúñiga, F. Muñoz, M. A. Márquez, E. S. E. Quesada, and L. R. G. Carrillo, "Load transportation using single and multiple quadrotor aerial vehicles with swing load attenuation," in Proceedings of International Conference on Unmanned Aircraft Systems (ICUAS), pp. 269-278, IEEE, Dallas TX, USA, June 2018.

[13] I. Palunko, P. Cruz, and R. Fierro, "Agile load transportation: safe and efficient load manipulation with aerial robots," IEEE Robotics \& Automation Magazine, vol. 19, no. 3, pp. 69-79, 2012.

[14] I. Palunko, R. Fierro, and P. Cruz, "Trajectory generation for swing-free maneuvers of a quadrotor with suspended payload: a dynamic programming approach," in Proceedings of 2012 IEEE International Conference on Robotics and Automation, Saint Paul, MN, USA, May 2012.

[15] B. S. Rego and G. V. Raffo, "Suspended load path tracking control using a tilt-rotor UAV based on zonotopic state estimation," Journal of the Franklin Institute, vol. 356, no. 4, pp. 1695-1729, 2019.

[16] M. A. Santos and G. V. Raffo, "Path tracking model predictive control of a tilt-rotor UAV carrying a suspended load," in Proceedings of the 2016 IEEE 19th International Conference on Intelligent Transportation Systems (ITSC), pp. 1458-1463, Rio de Janeiro, Brazil, November 2016.

[17] T. Lee, K. Sreenath, and V. Kumar, "Geometric control of cooperating multiple quadrotor UAVs with a suspended payload," in Proceedings of the IEEE Conference on Decision and Control, pp. 5510-5515, IEEE, Florence, Italy, December 2013.

[18] F. A. Goodarzi, D. Lee, and T. Lee, "Geometric stabilization of a quadrotor UAV with a payload connected by flexible cable," in Proceedings of the 2014 American Control Conference, pp. 4925-4930, IEEE, Portland, OR, USA, June 2014.

[19] M. E. Guerrero-Sánchez, D. A. Mercado-Ravell, R. Lozano, and C. D. García-Beltrán, "Swing-attenuation for a quadrotor transporting a cable-suspended payload," ISA Transactions, vol. 68, pp. 433-449, 2017.

[20] P. Li, L. Xiong, F. Wu, M. Ma, and J. Wang, "Sliding mode controller based on feedback linearization for damping of sub-synchronous control interaction in DFIG-based wind power plants," International Journal of Electrical Power \& Energy Systems, vol. 107, pp. 239-250, 2019.

[21] P. Li, J. Wang, F. Wu, and H. Li, "Nonlinear controller based on state feedback linearization for series-compensated DFIGbased wind power plants to mitigate sub-synchronous control interaction," International Transactions on Electrical Energy Systems, vol. 29, no. 1, p. e2682, 2019.

[22] L. Fridman and A. Levant, "Higher order sliding modes," in Sliding Mode Control In Engineering, pp. 53-101, Marcel Dekker Inc., New York, NY, USA, 2002.

[23] J. Guldner and V. Utkin, "The chattering problem in sliding mode systems," in Proceedings of the 14th International Symposium of Mathematical Theory of Networks and Systems, Perpignan, France, June 2000.

[24] H. Lee and V. I. Utkin, "Chattering suppression methods in sliding mode control systems," Annual Reviews in Control, vol. 31, no. 2, pp. 179-188, 2007.

[25] S. Yang and B. Xian, "Trajectory tracking control design for the system of a quadrotor UAV with a suspended payload," in Proceedings of the 36th Chinese Control Conference, pp. 777-782, IEEE, Dalian, China, July 2017.

[26] T. Yang, N. Sun, H. Chen, and Y. Fang, "Neural networkbased adaptive antiswing control of an underactuated shipmounted crane with roll motions and input dead zones," IEEE Transactions on Neural Networks and Learning Systems, pp. 1-14, 2019.

[27] N. Sun, D. Liang, Y. Wu, Y. Chen, Y. Qin, and Y. Fang, "Adaptive control for pneumatic artificial muscle systems with parametric uncertainties and unidirectional input constraints," IEEE Transactions on Industrial Informatics, p. 1, 2019. 


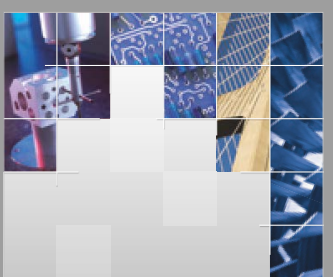

\section{Enfincering}
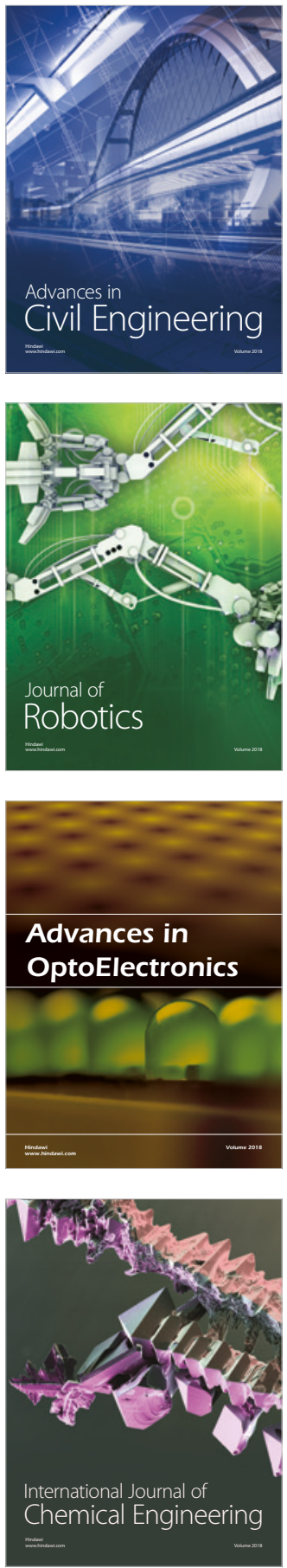

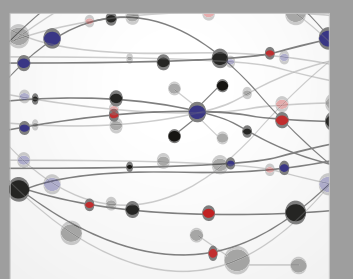

\section{Rotating \\ Machinery}

The Scientific World Journal

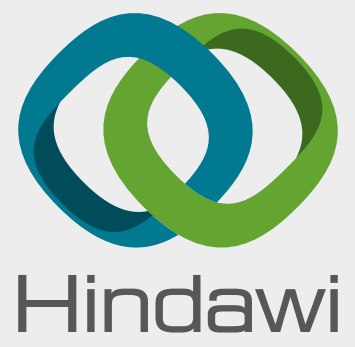

Submit your manuscripts at

www.hindawi.com
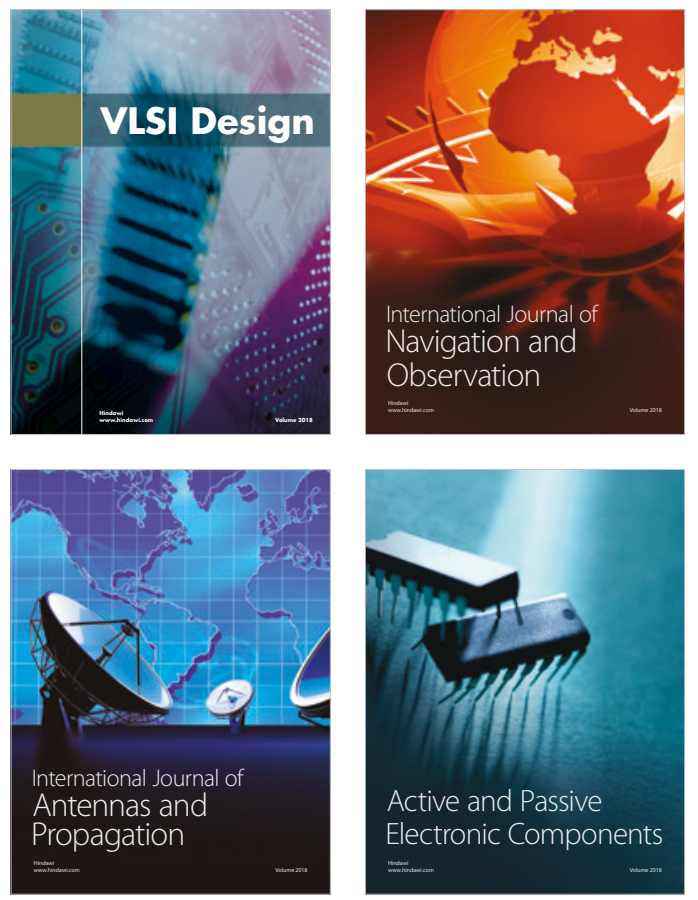
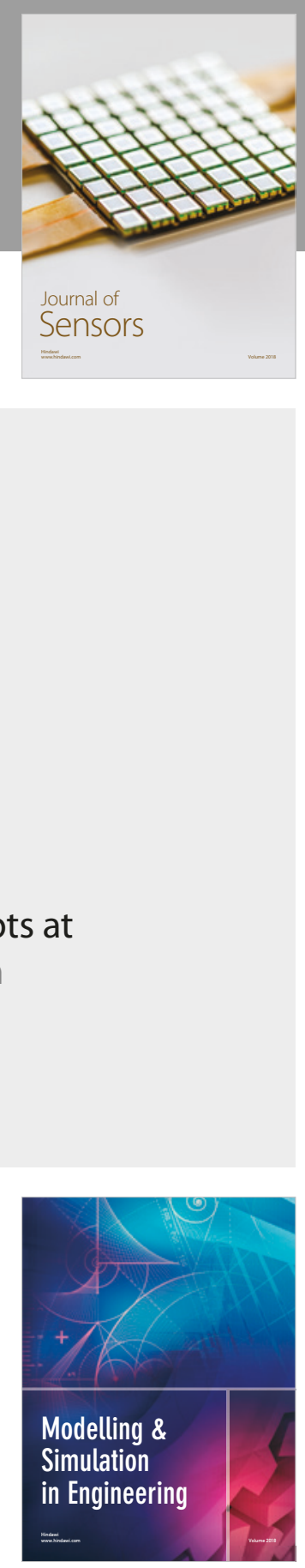

\section{Advances \\ Multimedia}
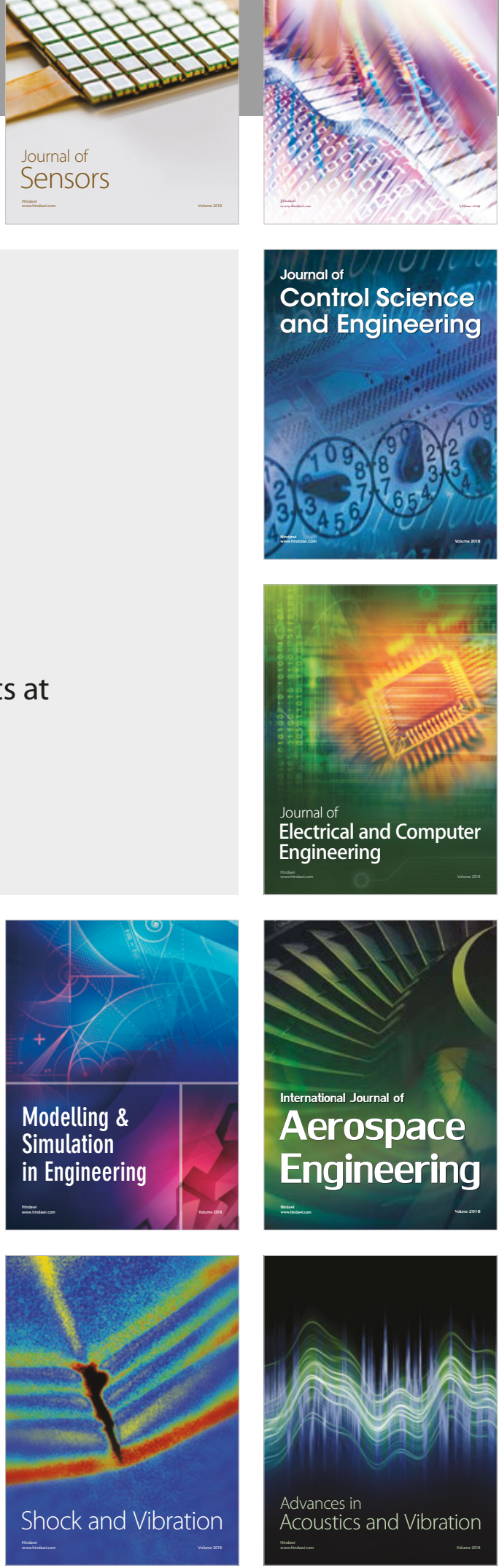